\title{
Introduction to the Special issue on Qualitative and Quantitative Research in Child and Adolescent Psychotherapy
}

\author{
Silvia Salcuni, ${ }^{1 \otimes}$ Claudia Capella, ${ }^{2}$ Adriana Lis ${ }^{1}$
}

In the last years it has been recognized that qualitative - as well as quantitative - research can inform clinical practice, making important contributions, and helping to bridging the gap between research and practice in psychotherapy. However, most psychotherapy research, both with quantitative and qualitative methodology has been done with adults, neglecting either the importance of empirical studies with children and adolescents and the special characteristics of psychotherapy in these age groups, which are sensitive to developmental issues (Midgley, 2004). In the recent years, it has been created a special interest group of Society for Psychotherapy Research on Child and Family Therapy Research (SPR-CaFTR), which aim is to improve the specificity of this field; SPR-CaFTR organized in the last SPR meetings different panels and discussions related to this topic, showing how qualitative and quantitative research in child an adolescent psychotherapy is growing. Despite this growing interest, it is difficult to find published papers using qualitative and quantitative research in child and adolescent psychotherapy.

Within their international collaboration, Claudia Capella and Adriana Lis proposed to the journal Research in Psychotherapy: Psychopathology, Process and Outcome (RIPPPO) a call for papers in this field to fill the gap found in literature, and they obtained the enthusiastic approval by the Editorial Board.

We are pleased to introduce the readers of RIPPPO to the rich final collection of 18 articles on research in psychotherapy on children and adolescents. In line with the journal scope, high quality papers from Sweden, Chile, Italy, Brazil, Argentina, and

\footnotetext{
${ }^{1}$ Department of Developmental and Social Psychology, University of Padua, Padua, Italy;

${ }^{2}$ Department of Psychology, University of Chile, Santiago, Chile.

Corresponding author: Silvia Salcuni, Department of Developmental and Social Psychology, University of Padua, via Venezia 8, 35131 Padua, Italy. Tel.: +39.049.8276544; E-mail: silvia.salcuni@unipd.it
}

USA, devised from different theoretical and methodological perspectives, were published. The special issue contains several kinds of contributions, divided into two RIPPPO volumes, 18(2) 2015 and 19(1) 2016: proposal of new methodology, original researches in psychotherapy on children and on adolescents, single case studies, multiple informants researches, and in conclusion a literature review.

The article collection starts with a very important work developed at the Institute of Psychotherapy in Stockholm, by Sandell (2015a, 2015b): the Manual and Commentary of Rating the outcomes of psychotherapy or psychoanalysis using the Change After Psychotherapy (CHAP) scales. It has been inspired by American studies with follow-up interviews after psychoanalysis by Pfeffer (1959) and Schlessinger and Robbins (1974), among others. CHAP manual is presented and one (or more) interviews as well as a set of ratings on the basis of this or these interviews are illustrated. These interviews are basically focused on the patient's subjective experience of the state of having changed or not. The power of the method is that it may be used both for research or more formal evaluation, and for the therapist once in a while systematically to judge for himself/herself how a therapy is developing.

If CHAP resulted useful both for adults and adolescents patients evaluation, Luzzi, Bardi, Ramos, and Slapak (2015) presented the preliminary result of a method of psychoanalytic psychotherapy process analysis specifically developed for children (MAPPP$\mathrm{C})$. Within the perspective of the object relations theory, changes in child patients' anxieties, defense mechanisms, object relations and unconscious phantasies, in each psychoanalytic psychotherapy session and throughout their entire psychotherapy were analyzed both from a qualitative and quantitative point of view. MAPPP-C indicators, units of analysis, development of analytical codes, training of raters and inter-rater reliability as well as examples of MAPPP$\mathrm{C}$ application were presented. 
Underlining the importance to improve non-traditional gathering information processes with children during psychotherapy, Capella et al. (2015) proposed a new methodology based on drawings combined with in-depth interview, in order to better understand how children view psychotherapy and psychotherapeutic change. Authors took into account non-verbal resources that better facilitate expression in developmental age and presented a single case analysis of the drawing of an eight year-old boy, evaluated pre and post treatment. This methodology provided crucial insight into the psychotherapy process, in respect with patient developmental needs.

To study adolescents' narratives resulted one of the best approach to gather information about the psychotherapy change. Beiza et al. (2015) examined narratives among five teenagers, during long-term residential care programs, who have successfully completed specialized psychotherapy after being sexually abused. Two different narrative positions regarding the meanings of healing after an experience of sexual abuse emerged, thought subjects' voices showing the possibilities for generating recovery narratives and suggesting important specific guidelines of therapeutic intervention.

Within the attachment theory framework, D'Onofrio, Pace, and Cavanna (2015) analyzed change in reflective functioning and attachment pattern after a psychotherapy of an anorectic adolescent, using the analysis of narratives of the Attachment Interview for Childhood and Adolescence (AICA) at pre and post-treatment. Interviews were coded both with the attachment coding system and the Reflective Functioning scale. AICA resulted a suitable method to assess the outcomes in adolescent psychotherapy, as well as the Adult Attachment Interview is useful in adult psychotherapy.

Moving to the filed of Q-sort methodology, Di Lorenzo, Maggiolini, and Suigo (2015) investigated, within a psychoanalytic approach, the typical features of Italian adolescence psychodynamic psychotherapy and its characteristics compared with other adolescence psychotherapeutic approaches, differentiating typical therapists' responses to adolescent patients. The Adolescent Psychotherapy Q-Set and the Therapist Response Questionnaire resulted very useful in defining how the therapeutic process was characterized by a positive and protective countertransference responses, with a priority to helping adolescent make sense of his own experience, focusing on present relationships and emotions rather than on past, highlighting strong similarities with mentalization based therapy approach.

Fernandez (2016) deepened the role of therapeutic alliance quality in the first sessions and therapeutic outcomes of adolescents' psychotherapy, putting in lights which perspectives (of the adolescents, therapists, or parents) and times of evaluation (first, second, or third session) more strongly influence outcomes. One of the strength of the study is its descriptive-correlational and naturalistic settings approach. The results showed the importance of the initial construction of the working alliance by both participants'- adolescents and therapist- perspectives.

An original contribution was provided by Carrasco (2016). Considering the big attraction new technologies and video games had on adolescents, a development and research project was implemented to design an online adventure video game as a psychotherapeutic tool for depressed adolescents. Five psychotherapists and fifteen of their female adolescent patients with symptoms of depression tested the game. The study suggested that future developments of video games for mental health are worthwhile and it provides some ideas on how to incorporate psychotherapeutic notions in ludic environments.

In developmental age is greatly important to gain a wide comprehension of patients' experience; the use of a multiple informant research design contributed to reach a wide amount of knowledge about the little patients and to improve their well being. Olhaberry, León, Seguel, and Mena (2015) implemented a brief intervention for mother-infant dyads with maternal depressive symptomatology using video-feedback intervention (VFI). The longitudinal, quasi-experimental qualitative study counted 61 mother-baby dyads. The results show VFI as an effective tool to improve the quality of the interaction, with a significant growth of maternal sensitivity, and a significant fall in pathological control, in the frequency of major depression episodes in mothers and in bonding risk.

Gatta, Sisti, Sudati, Miscioscia, and Simonelli (2016) contributed to the scientific debate about the effectiveness of assessment of interventions in infant mental health and presents the main results of one year of integrated treatments characterized by psychodynamic psychotherapy for the child/adolescent, parental support for the parents and the observation and assessment of family interactions, using the Lausanne Trilogue Play (LTP) as a new tool for the clinical assessment of interventions. Twenty-three children and adolescents, and their families were assessed longitudinally evaluating psycho-behavioral problems and family interactions. The results suggested a favorable outcome concerning a reduction of patients' symptomathology and stability in the family interaction modalities. The evaluation of the state of family interactions with LTP helped clinicians in focusing the intervention on those aspects that remain dysfunctional.

A growing body of attachment literature has focused on bridging the gap between research and clinical applications, even in work with adoptive families. Pace, D'Onofrio, Guerriero, and Zavattini (2016) analyzed a single case study with a lateadopted child and his adoptive mother. A brief-term clinical intervention focused on attachment relation- 
ships and representations was performed in the first year after placement. Results showed the utility of the attachment-intervention to help the mother enhance her sensitivity skills and her ability to understand both her own past attachment experiences and her child's insecure attachment as a consequence of the failures of his previous experiences of care.

Remaining on parents' functioning and influence on children and adolescents' development, Manfredi et al. (2016), highlighted how perceived parental criticism, perfectionistic concern over mistakes and ruminative brooding are powerful in predicting internalized self-criticism; in addition, they explored the predictive value all the considered variables for depressive mood in a non-clinical population. The findings suggested that a thinking style characterized by ruminative brooding and the tendency to be concerned with mistakes can facilitate the development of a self-critical person, more than a parental style perceived as critical. Moreover, the tendency to be self-critical may be more predictive of depressive symptoms than the other variables considered.

The call for qualitative and quantitative research in psychotherapy had been addressed fully by many clinical single cases, bringing very important contributions in the field of developmental psychopathology. Psychotics and autism spectrum disorder refers to a group of diseases determined by multiple conditions and primarily defined on the base of behavioral patterns. Standing the scarcity of studies evidencing the effectiveness of therapeutic and developmental approaches situated in a psychodynamic framework, the study by Ramires, Carvalho, Schmidt, Fiorini, and Goodman (2015) contributed to investigate the interaction structures of a 30months psychodynamic therapy process of a boy diagnosed with Asperger's disorder, using the Child Psychotherapy Q-Set. A mixed longitudinal study, based on the systematic case study procedure, was performed and the Rorschach method was used as the outcome measure.

Vecchiato, Sacchi, Simonelli, and Purgato (2016) proposed a qualitative research on the single-case intervention with an autistic adolescent, admitted to a therapeutic community, which bases its intervention on a specific psychodynamic approach. Authors highlighted the efficacy of the psychodynamic intervention by monitoring the therapeutic process, at the beginning of the treatment, after 6 and after 12 months. Results showed that the psychodynamic setting and the therapeutic intervention foster an improvement of adaptive behavior, such as communication, everyday life skills and socialization.

The recovery from for first-episode schizophrenia (FESZ), understood as a positive adaptation to the experience of psychosis, is closely connected with the possibility of the adolescent to re-embark on his/her biographical trajectory. Abarzua, Venegas, and Hidalgo (2016) put attention to the subjective experience of diagnosis and treatment in two adolescents with FESZ. The study performed semistructured interviews and qualitative content analysis in order to describe, from patients' perspectives, the subjective experiences associated with the processes of diagnosis and treatment of FESZ. The results suggested the family emotional climate associated with the experience of FESZ converge to the central phenomena of appropriation and resistance to the appropriation of FESZ in the patients: such findings highlight the relevance of taking the adolescent who has a FESZ into consideration as an active subject, going beyond an approach that traditionally has excluded them as agents of their diagnosis and treatment processes.

Two paper from different theoretical perspective, considered mood disorder in adolescence. An early adolescent, affected by a general anxiety disorder in a once a week psychoanalytically oriented psychotherapy, was assessed in respect with defense mechanisms trends and personality structure changes (Di Riso, Gennaro, \& Salcuni, 2015). Quantitative and narrative profiles of personality and defensive functioning were integrated; a log linear procedure and a multiple correspondence analysis were chosen to provide a map of the evolution of the patterns of defense mechanisms throughout the course of treatment. The study highlighted how nonintensive psychoanalytic psychotherapy can help early adolescents with clinical problems to improve their overall mental functioning, in terms of symptomatology but also of personality structure. The clinical utility of a brief cognitive-behavioral intervention was shown by Quezada Scholz (2016), who focused on confronting stress and reducing the anxiety-depressive symptomology in students of the Odontology Faculty. Using an A-B-C design, authors evaluated the changes in 5 single cases, using the OQ-45.2, the Dental Environment Stress Questionnaire and a semi-structured interview. Author's conclusion was that the dental students were benefitted by the intervention and recognized changes in their behavior and cognitions.

The last paper to this special issue is a literature review about a very unexplored theme: the impact of therapist's pregnancy on the child patient. Schmidt, Fiorini, and Röhnelt Ramires (2015) presented this original contribution, highlighting the big lack of knowledge in literature about pregnancy, one of the stronger catalyzer for the transference and/or for countertransference reactions.

From this collection of papers, we can have a very interesting picture of the state of art of psychotherapy research with children and adolescents, which shows how it is growing and including a variety of techniques, methodologies, theoretical orientations, treatment options for different disorders, and different ages of participants.

At the very end of this Editorial, after one year of 
very fruitful collaborations and work, a special remark goes to the Guest Editors Adriana Lis and Claudia Capella and to their will to offer their professionalism and experience in managing this special issue, to all the authors who agreed to participate in this project with their original contributions, and to all the reviewers, whose punctual comments, every time it was necessary, enhanced and promoted the quality of research and manuscripts within an interactive profitable revision process.

\section{References}

Abarzua, M., Venegas, F., \& Hidalgo, X. (2016). Subjective experience of diagnosis and treatment in two adolescents with first-episode schizophrenia. Research in Psychotherapy: Psychopathology, Process and Outcome, 19(1). doi: 10.4081/ ripppo.2016.189

Beiza, G., Capella, C., Dussert, D., Rodríguez, L., Águila, D., Gutiérrez, C., \& Lama, X. (2015). Institutionalized adolescents in therapy: Narratives of psychotherapy and healing from sexual abuse. Research in Psychotherapy: Psychopathology, Process and Outcome, 18(2), 62-71. doi: 10.7411/RP.2016.004

Capella, C., Rodriguez, L., Aguila, D., Dussert, D., Lama, X., Gutierrez, C., \& Beiza, G. (2015). Storied images of psychotherapeutic change: Approaching children's voices through drawings. Research in Psychotherapy: Psychopathology, Process and Outcome, 18(2), 141-151. doi: 10.7411/RP. 2015.109

Carrasco, A.E. (2016). Acceptability of an adventure video game in the treatment of female adolescents with symptoms of depression. Research in Psychotherapy: Psychopathology, Process and Outcome, 19(1). doi: 10.4081/ripppo.2016.182.

Di Lorenzo, M., Maggiolini, A., \& Suigo, V.A. (2015). A developmental perspective on adolescent psychoanalytic psychotherapy. An Italian study with the Adolescent Psychotherapy Q-Set. Research in Psychotherapy: Psychopathology, Process and Outcome, 18(2), 102-113. doi: 10.7411/RP. 2015.106

Di Riso, D., Gennaro, A., \& Salcuni, S. (2015). Defensive mechanisms and personality structure in an early adolescent boy: Process and outcome issues in a non-intensive psychoanalytically oriented psychotherapy. Research in Psychotherapy: Psychopathology, Process and Outcome, 18(2), 114128. doi: 10.7411/RP.2015.101

D’Onofrio, E., Pace, C.S., \& Cavanna, D. (2015). Qualitative research in adolescent psychotherapy: Attachment and Reflective Functioning as psychotherapy's outcomes of an adolescent with anorexia nervosa. Research in Psychotherapy: Psychopathology, Process and Outcome, 18(2), 93-101. doi: 10-7411/RP.2015.104

Fernandez, O.M. (2016). Therapeutic alliance in the initial phase of psychotherapy with adolescents: Different perspectives and their association with therapeutic outcomes. Research in Psychotherapy: Psychopathology, Process and Outcome, 19(1). doi: 10.4081/ripppo.2016.180.

Gatta, M., Sisti, M., Sudati, L., Miscioscia, M., \& Simonelli, A. (2016). The Lausanne Trilogue Play within the treatments' effectiveness evaluation in Infant Mental Health: A preliminary report. Research in Psychotherapy: Psychopathology, Process and Outcome, 19(1). doi: 10.4081/ripppo.2016.198.

Luzzi, A.M., Bardi, D., Ramos, L., \& Slapak, S. (2015). A study of process in psychoanalytic psychotherapy with children: the development of a method. Research in Psychotherapy: Psychopathology, Process and Outcome, 18(2), 7281. doi: $10.7411 /$ RP. 2015.110

Manfredi, C., Caselli, G., Pescini, F., Rossi, M., Rebecchi, D., Ruggiero, G.M., \& Sassaroli S. (2016). Parental criticism, self-criticism and their relation to depressive mood: An exploratory study among a non-clinical population. Research in Psychotherapy: Psychopathology, Process and Outcome, 19(1). doi: 10.4081/ripppo.2016.178.

Midgley, N. (2004). Sailing between Scylla and Charybdis: incorporating qualitative approaches into child psychotherapy research. Journal of Child Psychotherapy, 30(1), 89-111. doi:10.1080/0075417042000205814

Olhaberry, M., León, M.J., Seguel, M., \& Mena, C. (2015). Video-feedback intervention in mother-baby dyads with depressive symptomatology and relationship difficulties. Research in Psychotherapy: Psychopathology, Process and Outcome, 18(2), 82-92. doi: 10.7411/RP.2015.102

Pace, C.S., D’Onofrio, E., Guerriero, V., \& Zavattini, G.C. (2016). A proposal for a brief-term post-adoption intervention in the attachment-perspective: a single case study with a late-adopted child and his adoptive mother. Research in Psychotherapy: Psychopathology, Process and Outcome, 19(1). doi: 10.4081/ripppo.2016.197.

Pfeffer, A.Z. (1959). A procedure for evaluating the results of psychoanalysis: A preliminary report. Journal of the American Psychoanalytic Association, 7(3), 418-444. doi: 10.1177/ 000306515900700302

Quezada Scholz, V.E. (2016). A brief cognitive-behavioral intervention for stress, anxiety and depressive symptoms in students of odontology. Research in Psychotherapy: Psychopathology, Process and Outcome, 19(1). doi: 10.4081/ ripppo.2016.192.

Ramires, V.R., Carvalho, C., Schmidt, F.M.D., Fiorini, G.P., $\&$ Goodman, G. (2015). Interaction structures in the psychodynamic therapy of a boy diagnosed with Asperger's disorder: a single-case study. Research in Psychotherapy: Psychopathology, Process and Outcome, 18(2), 129-140. doi: 10.7411/RP.2015.112

Sandell, R. (2015a). Change After Psychotherapy (CHAP): un metodo di valutazione del cambiamento alla fine della psicoterapia. Psicoterapia e Scienze Umane, 49(4), 595-628. doi: 10.3280/PU2015-004004

Sandell, R. (2015b). Rating the outcomes of psychotherapy using the Change After Psychotherapy (CHAP) scales. Manual and commentary. Research in Psychotherapy: Psychopathology, Process and Outcome, 18(2), 32-49. doi: 10.7411/RP.2015.111

Schlessinger, N. \& Robbins, F. (1974). Assessment and follow-up in psychoanalysis. Journal of the American Psychoanalytic Association, 22(3), 542-567. doi: 10.1177/ 000306517402200305

Schmidt, F.M.D., Fiorini, G.P., \& Röhnelt Ramires, V.R. (2015). Psychoanalytic psychotherapy and the pregnant therapist: A literature review. Research in Psychotherapy: Psychopathology, Process and Outcome, 18(2), 50-61. doi: 10.741/ RP.2015.107

Vecchiato, M., Sacchi, C., Simonelli, A., \& Purgato, N. (2016). Evaluating the efficacy of psychodynamic treatment on a single case of autism. A qualitative research. Research in Psychotherapy: Psychopathology, Process and Outcome, 19(1). doi: 10.4081/ripppo.2016.194 